\title{
Lernen und die gesellschaftliche Reproduktion: Vier Thesen über die Wirksamkeit von Ideologie in der Schule
}

\begin{abstract}
Michael Brandmayr
Zusammenfassung

Der Beitrag beschreibt einige zentrale Überlegungen, unter welchen Voraussetzungen schulische Praktiken als Ideologien beschrieben werden können und in welchem Maße die Schule an der ideologischen Reproduktion der Gesellschaft beteiligt ist. Der Beitrag entwirft vier Thesen über die Wirkungsweise von Ideologie in der Schule, die sich der Frage einer ökonomischen Funktionalisierung von Lernen, des Sozialen bzw. der Sozialität in Formen der Subjektivierung sowie der Dialektik des Prozesses der ideologischen Vermittlung widmen. Diese Thesen werden auf Basis einer diskursanalytischen Untersuchung eines schulpädagogischen Leitbildes von idealem Lernen im österreichischen Schulsystem (Brandmayr 2017) entwickelt, deren Ergebnisse nach einer Diskussion des Ideologiebegriffs dargestellt werden. Ideologie wird in diesem Beitrag als wesentlich durch Praktiken vermittelt begriffen, in der Schule besonders durch die Praktik des Lernens. Diese Praktiken strukturieren Wahrnehmungs- und Interpretationsweisen und leiten Subjektivierungsvorgänge an. Über die Analyse des Lernens kann also gefragt werden, wie die Schule zur Vermittlung von Ideologien und damit zur Reproduktion der Gesellschaft beiträgt.
\end{abstract}

Schlagwörter: Diskurs um Schule, Lernen, Ideologie, Reproduktion, Subjektivierung

\section{Learning and the reproduction of societies:}

Four theses on the efficacy of ideology in schools

\section{Abstract}

The paper discusses, under which preconditions certain practices in schools can be described as ideologies and in which forms schools participate on the reproduction and distribution of ideologies in society. It elaborates four assumptions on the effectiveness of ideologies in schools, regarding the problems of an economic functionalism in modes of learning, the question of the social in processes of subjectivation and the dialectics in the distribution process of ideology. These assumptions are based on a discourse analysis that examined the guiding conceptions of ideal learning in the Austrian educational system currently in use (Brandmayr 2017). The findings of this analyzes are presented after a discussion of the term ideology. Ideology is therby understood as being distributed by practices, in schools this means mainly by learning processes. Those practices structure modes of perception and interpretation and guide processes of subjectivation. So by analyzing learning practices in schools, it can be questioned, how do schools contribute to the ideological distribution and therefore also to the reproduction of societies.

Keywords: discourse on school development, learning, ideology, reproduction, subjectivation

\footnotetext{
* Michael Brandmayr, Projektmitarbeiter am Institut für Erziehungswissenschaften der Universität Innsbruck. E-Mail: michael.brandmayr@uibk.ac.at.
} 


\section{Einleitung und Fragestellung}

Die Schule, so schreibt Louis Althusser (2010: 67, 68), „nimmt vom Kindergarten an Kinder aller sozialen Klassen auf und vom Kindergarten angefangen trichtert sie ihnen mit neuen wie mit alten Methoden jahrelang - in eben den Jahren, in denen das Kind am leichtesten ,verwundbar' ist, - [...]-,know-how' ein, das in herrschender Ideologie verkleidet (Französisch, Rechnen, Naturkunde, Wissenschaften, Literatur) oder aber ganz einfach die herrschende Ideologie im reinen Zustand ist (Moral, Staatsbürgerkunde, Philosophie)“. Eine etwas andere, jedoch in ihren Effekten nicht unähnliche Beschreibung liefert Siegfried Bernfeld (1973: 97), wenn er seinen fiktiven Unterrichtsminister Machiavelli Folgendes sagen lässt: „Die Kinder müssen die bürgerliche Klasse lieben lernen. Und dieser Unterricht muss so nachdrücklich, so sicheren Erfolgs sein, dass ein ganzes Leben in Not und Sklaverei verbracht, nicht hinreicht, diese Liebe zu verlöschen. Was in Wahrheit gewaltsam erzwungene Ausbeutung ist, wir wissen es, soll ihnen als freiwillig dargebrachtes Opfer der Liebe erscheinen. Sie sollen Mehrwert leisten, aber sie sollen es gern tun, aus innerem Liebeszwang, so wie der Liebhaber seiner Geliebten, der Gläubige seinem Gott opfert."

Seit 1925, dem Erscheinungsjahr von Bernfelds Schrift "Sisyphos und die Grenzen der Erziehung“, finden sich viele Darstellungen, welche die Rolle der Schule als Instanz der Vermittlung gesellschaftlicher Ideologien beschreiben. Darin finden sich unterschiedliche Auffassungen, was als Ideologien zu verstehen ist und welche schulischen Praktiken als Materialisation von Ideologien begriffen werden können. Jenseits dieser Auffassungsunterschiede eint die meisten dieser Ansätze, dass die Beschreibungen die gesellschaftliche Metaebene fokussieren und seltener versucht wird, Ideologie auf der Ebene alltäglicher Praktiken zu beschreiben, d. h. schulische Praktiken in historischideologiekritischer Perspektive zu analysieren. Daher versucht dieser Beitrag, eine Theorie über die Funktions- und Wirkungsweise von Ideologien in der Schule mittels einer Analyse der Praktik des Lernens, als der zentralen Tätigkeit in der Schule, zu geben. Dies unternimmt der Beitrag auf Basis einer diskursanalytischen Untersuchung von Leitbildern des Lernens im österreichischen Schulsystem, deren methodischer Aufbau in Abschnitt drei vorgestellt wird, und deren wesentliche Ergebnisse in Abschnitt vier kurz dargestellt werden. Im Hauptteil der Arbeit werden vier Thesen über die Funktion von Ideologie in ihrer Praxis ausgeführt.
Zunächst möchte der Beitrag jedoch wesentliche Auffassungen des Ideologiebegriffs knapp darstellen sowie das Verständnis darlegen, das der Theoriebildung im Folgenden zu Grunde liegt.

\section{Zum Begriff der Ideologie}

In den 1970er-Jahren prägte der Begriff des „heimlichen Lehrplans“ bzw. des „Hidden Curriculums“ die Debatte um die „anderen“, unbewussten Effekte der Schule (für einen Überblick siehe Brandmayr 2015). Vielfach findet sich hier jedoch ein Verständnis von Ideologie, wonach aus der Funktionsweise schulischer Strukturen sehr deterministisch auf die vermittelte Ideologie geschlossen werden kann. Die SchülerInnen würden zur Übernahme genau jener Regeln und Verhaltensweisen gezwungen werden, nach denen die Schule organisiert ist. Welche Normen, Werte und Einstellungen SchülerInnen also übernehmen, aber auch wie das vermittelte Wissen angewendet wird und wie alltägliche Interaktionen in der Schule strukturiert sind, all dies wird durch offene und latente Mechanismen in der Schule gesteuert, da die Schule Anpassung belohnt und diese zur Not auch mit physischer und psychischer Gewalt durchsetzt. Ideologie erhält hier eine totale, unausweichliche Dimension, die SchülerInnen sind in dieser Perspektive nur passive, rezipierende Objekte. Die Art der Ideologie bedingt sich dabei aus dem materiellen Unterbau der Gesellschaft - die Regeln der Schule, wie Leistungsbereitschaft oder Pünktlichkeit, stammen letztlich aus der Arbeitswelt. Bowles und Gintis (1976) beschreiben dies als das „correspondence principle“ zwischen Schule und Gesellschaft; der letztlich alles bestimmende Zweck der Schule sei die Vorbereitung auf die Arbeitswelt.

Die Studie „learning to labour" von Willis (1979/2013) konturiert jedoch einen anderen Begriff der Ideologie, der viel stärker kulturell geprägt ist. Willis Studie zeigt, dass die lebensweltliche Verarbeitung von Ideologien deutlich differenzierter vollzogen wird; besonders aber weist er darauf hin, dass auch diejenigen SchülerInnen, die vom System keine Chance erhalten und rebellisch gegen die vorherrschende schulische Kultur auftreten, die Ideologie dennoch aufnehmen und reproduzieren. Willis zeigt, dass sich die SchülerInnen der englischen Vorstadt ihr Versagen selbst zuschreiben und ihr Glaube an die Gerechtigkeit des schulischen Leistungsprinzips trotz der Erfahrung von Benachteiligung ungebrochen hoch ist - sogar noch höher als der der Mittelschicht. Was Willis damit 
beschreibt, ist der umgekehrte Effekt eines ideologischen Prozesses: Die „Lads“ der englischen Vorstadt konstituierten ihre Identitäten gerade in Abgrenzung zur schulischen Kultur. Sie positionierten „ihre“ Kultur und die damit verbundenen Werte - die Bedeutung körperlicher Arbeit, Vorstellungen von Männlichkeit und eines damit verbundenen Sexismus usw. - aus der Erfahrung von Minderwertigkeit und Ablehnung, die ihnen in der Schule vermittelt wurden. Sie waren daher keineswegs passiv; vielmehr identifizierten sie sich in positiver Weise mit ihren Lebensentwürfen, entschieden sich aus ihrer Perspektive bewusst für ihre Art zu leben und gegen die bürgerliche Kultur, die sie in ihrer Deutung dann „zu Recht“ ablehnten. Dennoch reproduzierten sie gerade dadurch eine bereits bestehende soziale Hierarchie.

Auf Basis einer Re-Lektüre der Schriften von Marx erarbeitet Althusser ein demgegenüber nochmals anderes Konzept der Wirkungsweise von Ideologie, in dessen Zentrum der Begriff der Anrufung steht. Die Schule wird als zentraler ideologischer Staatsapparat beschrieben, dem - in Anlehnung an Gramsci gesprochen - die Funktion der Organisation eines gesellschaftlichen Konsenses zukommt. Die Gesellschaft erscheint darin nicht als Resultat konflikthafter Auseinandersetzungen sozialer Gruppen mit widerstrebenden Interessen, sondern die bestehende Ordnung wird als natürlich präsentiert, und zugleich werden dem Individuum bestimmte Positionen in dieser Ordnung angeboten. Wenn Althusser schreibt, Staatsapparate "lehren eine bestimmte Art von, know-how', aber in solchen Formen, dass dadurch die Unterwerfung unter die herrschende Ideologie oder die Meisterschaft in ihrer praktischen Ausübung [pratique] gesichert werden" (Althusser 2010: 43), meint er damit einen zentralen Aspekt seiner Ideologietheorie, nämlich, dass die schulische Praxis und die an ihnen mitwirkenden Subjekte Ideologien gemeinsam konstituieren. Die Ideologie ist der Praxis also nicht vorgängig, Ideologie nicht determinierend; dennoch gibt es kein Außerhalb der Ideologie, da die Praxis - durch das Moment der Anrufung - Individuen erst zu Subjekten macht. Der Moment, in dem die Lehrperson den/die SchülerIn als SchülerIn anspricht, ist ein konstitutiver Akt, der die Subjekte erst herstellt. Da das Subjekt aber nur in genau der konkreten, in der Anrufung vorgegebenen Ordnung existieren kann, sind Subjekte dieser Ordnung immer schon unterworfen - Ideologie ist produktiv und repressiv zugleich.

Die skizzierten Verwendungen des Ideologiebegriffes divergieren also insbesondere hinsichtlich jenes
Aspektes, ob Ideologie als „notwendig falsches Bewusstsein" (Rehmann 2004: 720) aufgefasst wird. Ein solches Verständnis findet sich im Verständnis von Ideologie, wie es besonders von der Frankfurter Schule verwendet wurde. Sie schließen dabei an das Bild von Marx und Engels an, welche die Metapher der Camera obscura prägten, wodurch den Menschen ihre Verhältnisse „auf den Kopf gestellt erscheinen“ (Marx/Engels 1958: 22). Ideologie bedeutet für die Frankfurter Schule das Wirken eines umfassenden Verblendungszusammenhangs, in dem die Menschen von der Kulturindustrie systematisch manipuliert werden. Das Individuum nimmt Ideologie in diesem Verständnis bloß passiv auf, ideologische Gehalte wirken umfassend und total - wobei sowohl stillschweigend festgelegt scheint, was Ideologie bedeutet und woher sie kommt, ohne dass dies je expliziert wird. Dies verleitet nicht nur zu apodiktischen, letztlich nicht kritisier- oder widerlegbaren (und daher selbst wieder ideologischen) Annahmen: In dieser Theoretisierung des Ideologiebegriffs findet sich auch kein Ausweg aus der Ideologie.

Anders ist dies bei Althusser und Laclau, für die Ideologie nicht einfach auf eine Bewusstseinsform reduziert werden darf, sondern über eine eigene Materialität verfügt. Ideologien sind - diskurstheoretisch gefasst - Objekte bzw. Objektivationen aus Diskursen, die eine konstitutive Funktion für Subjekte einnehmen. Für Laclau besteht das Wirken der Ideologie in ihrem Angebot kollektiver Repräsentationen von sozialen Identitäten, genauer gesagt in der ideologischen Formierung dieser Repräsentationen. Sie sind ideologisch, weil sie die (faktisch unmögliche) Illusion einer geschlossenen Gemeinschaft symbolisieren, in der Widersprüche zwischen Mitgliedern der Gemeinschaft überwunden werden. Dies geschieht, indem rund um den zentralen Signifikanten einer kollektiven Repräsentation (d. h. Begriffe wie „Volk“ oder "Nation“) Äquivalenzketten mit spezifischen Bedeutungen arrangiert werden, wodurch diese Signifikanten eine ideologische Bedeutung mit identifikatorischem Charakter erhalten. Ideologie dient also letztlich der Integration des Subjekts in die ökonomischen Verhältnisse der Gesellschaft - über die Schule - und der Reproduktion der gesellschaftlichen Produktionsverhältnisse. Ideologie bedeutet bei Althusser (2010: 78) das „imaginäre Verhältnis“ der Individuen „Zu den realen Verhältnissen, unter denen sie leben“. Dieses Verhältnis wird aber durch die „Praxis des ideologischen Wiedererkennens“ (ebenda) von den Individuen selbst mitkonstituiert, indem sie in ritualisierten, materialisierten Handlun- 
gen und Praktiken zu bestimmten Subjektpositionen finden und diese einnehmen. Alle Praxis ist diskursiv, d. h., dass Diskurse Praktiken organisieren und Ideologie wesentlich durch und in diesen Praktiken weitergegeben wird. Jedoch finden sich auch bei Althusser und Laclau/Mouffe nur wenige Analysen der unmittelbaren, lebensweltlichen Praxis des Subjekts.

Dieser Beitrag soll daher am Beispiel des Lernens darstellen, wie Mechanismen der Verzerrung, Verkennung und Verfremdung Praktiken so neu zusammensetzen, dass eine Reproduktion von Ideologien stattfindet. Dieser Beitrag möchte also darüber Auskunft geben, wie in Praktiken Ideologie vermittelt wird und ideologische Deutungen Gültigkeit erlangen. Lernen wird als Praktik verstanden, worin subjektive Aneignungsprozesse von Ideologien in aktiver Weise stattfinden, die eben nicht determiniert sind. Denn wie bei Althusser und Laclau und im Unterschied zur Frankfurter Schule unterliegen institutionelle Praktiken der diskursiven Formierung und damit dem hegemonialen ideologischen Deutungsangebot; jedoch bietet sich darin auch die Möglichkeit des Widerspruchs und damit eines Ausweges aus der Ideologie. Die darin eröffneten Spielräume der Performanz von Praktiken wie auch die individuelle Aneignung der Deutungsangebote in Praktiken sind für die erfolgreiche Vermittlung von Ideologien womöglich sogar ganz entscheidend; sie dienen zugleich der Identifikation mit der angebotenen Subjektposition, wie sie jedoch auch brüchige Linien darstellen, durch die das Subjekt in der Lage sein kann, sein imaginäres Verhältnis und dessen Widerspruchsfreiheit zu überwinden und die tatsächlichen gesellschaftlichen Verhältnisse zu erkennen.

\section{Methodologische Überlegungen einer ideologiekritischen Untersuchung von Lernpraktiken}

Die meisten gegenwärtigen Ansätze in der Schulpädagogik nähern sich dem Gegenstand des Lernens mit Methoden der Beobachtung und Befragung (für einen Überblick über die gegenwärtige Lerndebatte vgl. Faulstich 2014). Im Gegensatz dazu beschreibt diese Arbeit schulisches Lernen daher als diskursiv formierte soziale Praxis. Diskurse - dies ist die zentrale Prämisse dieses Beitrags - legen fest, was von AkteurInnen als ideales Lernen verstanden wird, woraus sich Deutungen, Wahrnehmungsweisen, aber auch ein konkretes Handlungswissen für Praktiken in der Schule ableiten. Diskursiv wird ein Leitbild von idealem Lernen formiert, das gewisse Deutungen der Art enthält, wie, was und wozu gelernt werden soll. In der diskursiven Auseinandersetzung wird festgelegt, was als effektive Verfahren und Methoden des Lernens, wünschenswerte Ziele, notwendige Bedingungen (wie „Spaß“ oder „Freiraum“), strukturelle Voraussetzungen in Institutionen (wie „Individualisierung“) u. v. m. gelten. Die Stärke des hier gewählten diskurstheoretischen Zugangs besteht gegenüber empirischen Einzelfallstudien darin, die gesellschaftliche Bedingtheit schulischer Praktiken, die ökonomischen und sozialen Interferenzen in Lernprozessen unmittelbar benennen und berücksichtigen zu können.

Die Formulierung des idealen Lernens verweist dabei auf ein notwendiges, aber unbestimmbares Konstrukt. Die Frage, was ideales Lernen wirklich ist, ist somit in ihrem Kern nicht beantwortbar - diesen Anspruch erhebt dieser Beitrag auch nicht. Es soll dagegen das gegenwärtig hegemoniale Deutungsangebot dargestellt werden, und darauf basierend sollen Praktiken des Lernens rekonstruiert und Subjektpositionen und Subjektivierungsweisen nachgezeichnet werden.

Diskurse wirken vor allem über die Produktion eines kollektiven Wissensvorrates in die Praxis, der den Referenzrahmen für Praktiken des Lernens bildet und dadurch bestimmte wünschenswerte Verhaltensmuster konturiert. Im diskursiven Feld der Schulpolitik verbinden sich dabei formal-juristische Mittel der Durchsetzung der Wissensordnung - über Lehrpläne, Verordnungen, Rundschreiben oder Aus- und Weiterbildungsprogramme - mit Mechanismen des diskursiven Ausschlusses, die über das Moment der Anrufung normierend wirken. Denn der Diskurs produziert auch Deutungen einer/s professionellen Akteurs/in, der/die als normativer Referenzpunkt für individuelle Optimierungsbestrebungen gilt und der/die abweichendes Verhalten als unprofessionell ausschließen kann: Im Konsens über ideales Lernen entstehen zugleich Leitbilder z. B. der idealen Lehrpersonen, die dieses Lernen anleitet, oder des/r idealen Schülers/ in, der/die richtig lernt. In Anlehnung an Keller kann dies als "professionsspezifische Identität“ bezeichnet werden, die auf Basis des diskursiv konstituierten Wissensvorrates gebildet wird und über die Anrufung als professionell zu Handelnder die Anwendung dieses Wissensvorrates überwacht: Der Satz „Eine richtige Lehrerin würde das nicht tun" erinnert eine Lehrerin daran, dass "genau sie“ als Lehrerin gemeint ist und zugleich aber nur dann damit gemeint ist, wenn sie 
sich auf diese und jene Art (nicht) verhält. So wird sie an die Möglichkeit des Ausschlusses aus ihrem Berufsstand erinnert, denn sie wäre keine „richtige“ Lehrerin mehr, wenn sie die normativen Vorgaben nicht anerkennt. Zugleich sorgt die Aussage dafür, dass die Wissensordnung einmal mehr aktualisiert wird und Gültigkeit behält.

Um die ideologische Wirkung von schulischen Praktiken nachzuzeichnen, muss also zunächst bestimmt werden, was ideales Lernen gegenwärtig bedeutet und wie sich ein Leitbild von idealem Lernen diskursiv konstituiert hat. Dies habe ich im Rahmen einer Analyse von 233 Dokumenten, die zur Neugestaltung der Schulpraxis im Zeitraum der vergangenen 15 Jahre angefertigt wurden, unternommen (vgl. ausführlich Brandmayr 2017). Diese beinhalten Gesetzesvorlagen, Verordnungen, Lehrpläne, Schulaufsichtsberichte, Leitlinien und Programmberichte von Unterrichtsbehörden, Rundschreiben, Erlässe, Weisungen; aber auch Dokumente aus der Aus- und Weiterbildung von LehrerInnen, (wissenschaftliche) Handreichungen und Unterrichtsbroschüren wurden berücksichtigt. Ausgangspunkt der Untersuchung war die Beobachtung einer gesteigerten Reformaktivität im österreichischen Bildungswesen in den vergangenen Jahren. Seit etwa 2007 lässt sich eine Steigerung von bildungspolitischen Umsetzungsmaßnahmen beobachten, wie auch die Aussage einer Dringlichkeit bzw. eines nationalen Bildungsnotstandes nachzuweisen ist. Unter dem Begriff der Bildungsreform wurde in Österreich mittels innerorganisatorischer und schulexterner Maßnahmen (Bildungsstandards, Einführung der Neuen Mittelschule als Regelschule, Matura Neu, Schulqualität Allgemeinbildung (SQA) und Reform der Schulaufsicht, neues LehrerInnendienstrecht, Reform der LehrerInnenausbildung, Modularisierung der Oberstufe und weitere) ein neues pädagogisches Leitbild von idealem Lehren und Lernen generiert und implementiert.

Die Untersuchung bedient sich der wissenssoziologischen Diskursanalyse (kurz WDA) als Forschungsprogramm. Der Diskurs um ein neues schulpädagogisches Leitbild von idealem Lernen wird mittels der Klassifikation von drei Diskussträngen - „Freude am Lernen“, „Individualisierung und Chancengerechtigkeit“ sowie „Bildungsstandards und Kompetenzorientierung“ analysiert. Die Diskursstränge wurden jeweils aus einem primären Datenkorpus generiert, der den Ausgangspunkt für die Untersuchung bildet. Dieser besteht aus Dokumenten, die sich in unmittelbarer, regulierender Weise auf das Praxisfeld der Schule beziehen - also juristische und behördliche Dokumente. Zunächst wurde dabei jeder einzelne Diskursstrang für sich analysiert, d. h. zentrale Elemente eines Deutungsmusters analysiert und identifiziert.

Im zweiten, nun rekonstruktiven Schritt wurde eine historische Analyse des Diskursverlaufs unter Rückgriff auf das Akteurskonzept der WDA unternommen, die auf Basis gemeinsamer Deutungsmuster klassifiziert wurden. Dabei wurde versucht, einzelne Aussage- und Deutungselemente von Akteursgruppen an den historischen Diskursverlauf rückzubinden, um Prozesse der Verknappung und Modifikation von Aussagen und Deutungen nachvollziehen zu können. Daraus sollen Hypothesen über die Konstitutionsprozesse der gegenwärtig dominanten Aussageelemente und ihnen zugrunde liegende Deutungen gewonnen werden.

Im dritten Schritt wurden die in den einzelnen Diskurssträngen ermittelten Deutungsmuster aufeinander bezogen. Erst daraus wurde versucht, ein gegenwärtiges pädagogisches Leitbild von idealem Lehren und Lernen zu bestimmen. Dieses etwas komplexe Vorgehen ist dem Datenkorpus und dem Diskursverlauf geschuldet: Es zeigte sich, dass vielfach Debatten über partikuläre Reformen von Schulentwicklung geführt werden, selten aber über ein grundsätzliches Leitbild, und daher der Diskurs aus verschiedenen Dokumenten zusammengesetzt werden musste. Das Vorgehen in diesem Schritt ist daher wesentlich interpretativ: Es gilt, im Diskurs "Gemeintes“, aber nicht Expliziertes, kenntlich zu machen, d. h. hintergründige Annahmen und Deutungen über ideales Lernen zu identifizieren und zu benennen.

Zentrales Werkzeug der Interpretation ist das schon erwähnte Konzept des Deutungsmusters (Keller 2014). Der Begriff verweist dabei auf den typisierbaren Gehalt von Aussagen und referenziert auf ihre Scharnierfunktion zwischen Diskurs und Subjekt. Er ist zu verstehen als ein ",Brückenkonzept', das gerade erlaubt, den Weg von der Diskursebene zur alltäglichen Deutungs- und Lebenspraxis zu gehen bzw. die Verflechtungen, Adaptionen, Aneignungen und Zurückweisungen im Deutungsmustergebrauch hier und da in den Blick zu nehmen" (Keller 2014: 155). Der Begriff erlaubt, auf immanente Normen, Werte und Annahmen hinter dem aktuellen schulpädagogischen Leitbild von idealem Lernen zu schließen sowie auf Positionen von Subjektivität, zu deren Einnahme die institutionelle Praktik des Lernens anleitet. 


\section{Was bedeutet gegenwärtig ideales Lernen?} Kurze Darstellung der Ergebnisse einer Analyse des gegenwärtigen schulpädagogischen Leitbildes von Lernen

Die folgende Darstellung bietet einen Überblick über zentrale Ergebnisse, die in der Analyse von Deutungsmustern des idealen Lernens ermittelt wurden. Es lassen sich zwei zentrale Deutungen nachweisen:

\subsection{Entgrenzung und Hybridität des Lernens}

Ein erstes Deutungsmuster lässt sich mit der Formulierung „Lernen als entgrenzte Tätigkeit“ beschreiben, in welchem ideales Lernen als von allen strukturellen und institutionellen Limitationen freigesetzt gedacht wird und sich vollkommen auf das individuelle Subjekt beziehen soll. In vielen Aussagen wird Lernen als ,immerwährende, eigenständige, höchst persönliche Aktivität jedes Menschen" (SQA 2012: 5) beschrieben und dabei so gedeutet, dass die Besonderheit des Prozesses, und somit auch ihre Bedingung, in der ungehinderten Individualität des Einzelnen besteht. Diese Aussagen beschreiben ideales Lernen daher als möglichst freies, unreguliertes Lernen, weil jeder Lernprozess anders verläuft - nur in der Berücksichtigung dieses Faktums kann in idealer Weise gelernt werden. Diskursiv wird dabei die Abschaffung einer jeden Regulierung mit der potenziellen Grenzenlosigkeit von Lernen verknüpft: „Infinite Learning“, der Untertitel der Online-Lernplattform OLAT der Universität Innsbruck, bringt das Deutungsmuster eines entgrenzten und daher potenziell grenzenlosen Lernens dabei auf den Punkt. Konkret impliziert dies:

- Eine Freisetzung von Lernen aus örtlichen Zusammenhängen bzw. festen räumlichen Vorgaben. Dabei steht die Aussage im Mittelpunkt, dass in künftigen Schulformen das Klassenzimmer kein reiner Lernraum, sondern auch ein Lebensraum sein kann - ebenso wie zu Hause auch gelernt werden kann.

- Eine Freisetzung von Lernen aus zeitlicher Regulierung: Sie besteht einerseits in der Fokussierung auf Aufgaben (und nicht zeitlicher Vorgaben) im Zuge der Individualisierung des Lernens, d. h. einer Orientierung an offenen Unterrichtsformen, die über keine fixierten Zeitabläufe verfügen, sondern die eigenverantwortliche Zeiteinteilung betonen.

- Eine Freisetzung des Lernens aus curricularen Grenzziehungen. Sie zeigt sich einerseits in einer Entgrenzung aus fachlich-disziplinären Zusammenhängen in einer Betonung von interdisziplinärem, verknüpfen- dem Lernen; zum anderen in einer Verknüpfung praktischer Orientierung des Lernens, das mehr auf den Transfer von Wissen, die Anwendung des Gelernten abzielt. Damit einhergehend erfolgte eine Entgrenzung aus diskursiv erzeugten Hierarchisierungs- und Kanonisierungstendenzen, die definierten, was als wichtige oder nützliche Bildungsinhalte von der Schule gelehrt werden sollten.

- Die Freisetzung des Lernens aus klar definierten biografischen Start- und Endpunkten. Das Postulat des lebenslangen Lernens setzt Zieldimensionen (man hat nie mehr ausgelernt) als auch biografische Grenzen (man ist nie mehr ,zu alt", um Neues zu lernen oder sich beruflich weiterzubilden) außer Kraft.

- Eine diskursive Entgrenzung, was ideale oder wünschenswerte Lernprodukte darstellen können. Auch dies stellt eine Folgerung aus der Aussage dar, auf die individuellen Begabungen und Stärken von SchülerInnen einzugehen. Lernprodukte sollen in erster Linie die individuellen Bestrebungen und die Kreativität von SchülerInnen reflektieren und sich weniger an definierten Vorgaben orientieren.

Um diese neuen Praktiken des Lernens zu realisieren bzw. weil diese Formen des Lernens einander bis zu einem gewissen Punkt widersprechen und doch nebeneinander existieren können, muss ideales Lernen als hybrides Lernen beschrieben werden. Der Begriff der Hybridität beschreibt hier, dass im neuen Leitbild zwei Formen von Lernen verbunden wurden, die im historischen Diskurs als unvereinbar gegolten haben. Er formuliert, dass in der Schule zugleich ein Lernen für den institutionalisierten Bildungsverlauf wie auch ein Lernen aus persönlich-praktischen Motiven stattfinden kann und soll. Damit wird einer Annahme widersprochen, die im historischen Diskurs durchgängig nachzuweisen ist - sie manifestiert sich etwa in Senecas Formulierung "non vitae, sed scholae discimus" bis zur gegenwärtigen Forderung nach mehr Lebenspraxis in der Schule: Beides meint, dass das Lernen in der Schule immer ein Lernen für die Schule darstellt und nicht fürs Leben oder für das Individuum. Schulisches Lernen hat mit persönlichem Lernen wenig zu tun, sondern wird als lebensfremd, fremdbestimmt und äußerlich gedeutet.

Das hybride Lernen überwindet diese diskursive Grenzziehung in der Aussage, das Lernen finde immer für das Individuum statt und dementsprechend bestimme nicht die Zieldimension die Lernweise (und davon abgeleitet auch nicht der Stoff, die Lernumgebung oder die Art der Prüfung), sondern das lernende Individuum. In dieser Deutung sei der Lernverlauf von der 
Art der Aneignung eines Stoffes durch das Individuum abhängig; das Individuum wird zur obersten Instanz, die über die Bedeutung von Inhalten, ihrer Verwendung und der Lernziele entscheiden kann und damit zum einzig möglichen Bestimmungspunkt für die Art des Lernens. Das Individuum entscheidet, welche Motive für Lernen entstehen und wie dementsprechende Lernverläufe $\mathrm{zu}$ gestalten sind. Damit werden pauschalierende Klassifikationen von Lernen (fremd- oder selbstbestimmt; eigene oder äußerliche Lernmotive; „berufliches“ und „persönliches“ Lernen etc.) obsolet und es kann somit nur noch von Lernen als allgemeinem Begriff gesprochen werden.

Ideales Lernen als möglichst entgrenzte Tätigkeit bedeutet - darin liegt eine der Paradoxien der neuen Formation - zwar formal mehr Möglichkeiten, aber dennoch keinen größeren Spielraum von möglichen Interpretationen (und damit eine größere Freiheit von schulischen AkteurInnen in der Gestaltung von Lernsettings), wohin Lernprozesse letztlich führen sollen. Ganz besonders dann nicht, wenn es darum geht, sie nicht wahrnehmen zu wollen. Denn das neue Leitbild von idealem Lernen impliziert zugleich einen Aufforderungscharakter $\mathrm{zu}$ einem spezifischen Gebrauch der Lernmöglichkeiten; so ist beispielsweise im Zuge der Kompetenzorientierung als neuem Unterrichtsparadigma die Verbindung von Lehrinhalten und ihrer Anwendungsmöglichkeiten in der Lebenspraxis von SchülerInnen nicht als neue Möglichkeit, sondern als Aufforderung zu verstehen. Entsprechend existiert ein Rechtfertigungsdruck von Lehrpersonen, die dies in ihren Lernarrangements nicht berücksichtigen, ebenso wie bei der Wahl von Themen, die nur einen geringen Bezug zur Lebenspraxis aufweisen. Aus der Möglichkeit, Lernverläufe zugunsten subjektiver Interessen und Dispositionen individuell(er) zu gestalten, entsteht also eine normative Aufforderung, dies zu tun, indem individualisiertes Lernen diskursiv als dominantes pädagogisches Leitbild positioniert wurde: „Individualisierung als Imperativ" (Sertl 2008). Dementsprechend kann die Formulierung der „Entgrenzung von Lernen“ auch verstanden werden als Beitrag einer Naturalisierung von Handlungsentscheidungen. Im Zweifel scheint es besser, zu lernen als nicht zu lernen, und es scheint vernünftiger, effizient zu lernen als ineffizient.

\subsection{Lernen, Leistung und der Prozess der Formung persönlicher Eigenschaften}

Wenn Lernen als höchstpersönlicher Prozess gedeutet wird, bezieht sich diese Deutung weniger auf den
Aspekt einer notwendigen Autonomie des Lernenden, d. h. Freiraum im Sinne einer Abwesenheit von verbindlichen Vorgaben. Im Anschluss an den zuletzt ausgeführten Gedanken, dass tatsächlich weniger formale Regulierungen existieren, aber die faktische Freiheit des Lernenden trotzdem nicht zugenommen hat, soll nun dargestellt werden, auf welche Art und Weise sich die Formen der Lernkontrollen und Leistungsbeurteilung zwischen Lehrpersonen und SchülerInnen verändert haben. Viele Änderungen in Form von Kontrolle und Beurteilung hängen damit zusammen, dass sich Lernen nun auf die Herausbildung persönlicher Eigenschaften bezieht, für deren Erfolg spezielle Rahmenbedingungen als notwendig erachtet werden.

Eine Vielzahl von Dokumenten nennt dabei die Herausbildung persönlicher Eigenschaften als zusehends bedeutsame Aufgabe von Schule in Hinblick auf die Notwendigkeit, in späteren Bereichen des Lebens über bestimmte Eigenschaften zu verfügen. Die ehemalige Bildungsministerin Claudia Schmied (2008: 14) meinte etwa: „Die Schule nimmt immer mehr die Rolle eines Lebensraumes ein, der eine Vorbereitung für zukünftige Lebensräume darstellen muss. Wir brauchen Menschen mit Eigenschaften. "Doch was ist hier gemeint und von welchen Eigenschaften spricht sie?

Einerseits werden hier personale und soziale Kompetenzen angesprochen, die im Diskurs oftmals als "Soft Skills“ bezeichnet werden, wie organisatorische Kompetenzen, das Arbeiten in Teams, Kommunikationsfähigkeiten, Feedback- und Rückmeldetechniken und dergleichen. Andererseits sind damit aber auch persönliche Tugenden, charakterliche Eigenschaften und gesellschaftliche Werte und Einstellungen gemeint. Diese sind sowohl expliziter Gegenstand von Unterricht oder werden als Teil des Erziehungsauftrages verstanden; sie sollen aber auch implizit in die Struktur von Lernsettings einfließen, um Lernprozesse effizienter $\mathrm{zu}$ machen und Interesse und Freude am Lernen fördern. Sowohl Soft Skills, als auch charakterliche Eigenschaften werden in Dokumenten als Gegenstände schulischen Lernens positioniert, und zwar gleichwertig zu konkreten, fachlichen Zielen und Kompetenzen.

Als zentralste Eigenschaft, die beiden Zielen dient - der Effizienz des Lernens und der Persönlichkeitsentwicklung -, kann Eigenverantwortung genannt werden. Es findet sich vielfach die Aussage, dass eigenverantwortliches, individualisiertes Lernen effizienteres Lernen darstellt. Eigenverantwortung schafft zudem die Basis, dass weitere wichtige Eigenschaften - Verantwortungsbewusstsein, Leistungsbereitschaft 
und Freude am Lernen, um weitere wichtige zu nennen - überhaupt gelernt werden. Denn der Lernprozess wünschenswerter Eigenschaften kann nicht standardisierbar erfasst und überprüft werden, sondern die Zieldimension von personalen Lernprozessen muss in Abhängigkeit von der Persönlichkeit des Lernenden getroffen werden. So lässt sich eine hohe Zahl von Aussagen nachweisen, die als Aufforderung an SchülerInnen zur „Arbeit an sich selbst“ zu verstehen sind. Die Analyse konnte zeigen, dass in diesen Dokumenten von einem arbeitstätigen Idealsubjekt ausgegangen wurde, welches an der Formung seiner Selbst tätig wird und sich dabei in seiner Gesamtheit - in körperlichen, emotionalen, sozialen, charakterlichen Dimensionen - bearbeitet. Dies ist für Prozesse der Subjektivierung folgenreich.

Wexler (2000) hat für das amerikanische Bildungssystem schon vor einigen Jahren beschrieben, wie im Zuge eines Wandels institutioneller Leitbilder die Identität der Schülersubjekte zusehends stärker an die erbrachte Leistung gebunden wird. Im amerikanischen Schulsystem werden SchülerInnen nicht mehr qua ihres Schulbesuches bereits als SchülerInnen anerkannt, sondern als imperfekte, mangelhafte Subjekte erachtet, die an sich arbeiten müssen, und nur dann „richtige“ SchülerInnen sind. Mangelhaft gelten SchülerInnen daher primär in moralischer Hinsicht (und nicht in kognitiver oder physischer), wenn sie nicht leistungsbereit sind, wodurch SchülerInnen in eine defensive Position gedrängt werden. Das Leitbild der Schule ist das eines imperfekten Subjektes, dessen Arbeit an sich selbst zu einer moralischen Pflicht avanciert: „Vom ersten ,Hallo, das uns sagt: ,Wir sind nicht so, wie du zu wissen glaubst,' bis zum letzten,Good-Bye, das die Welt daran erinnern soll, dass,Wir als Menschen wertvoll sind; arbeitet der Schüler daran, ein sichtbares, differenziertes und respektvolles Selbst zu schaffen. Die moralische Sprache des Gut- bzw. Anständigseins ist die Art und Weise, wie das Soziale in der entmarginalisierten Selbst-Existenz repräsentiert wird. Was auch immer sie in Bezug auf ihren Selbstwert vom Beginn ihrer Schulkarriere an zu beweisen haben, wird zugespitzt durch die Organisation der Schulen, die auf der Annahme basiert, dass das, was den Schülern fehlt, ein anständiges, moralisches Selbst ist." (Wexler 2000: 47f.)

Die Fokussierung auf persönliche Eigenschaften hat also zur Folge, dass in der schulischen Anrufung ein Subjekt mit ganz bestimmten, aber noch zu erbringenden Eigenschaften konstituiert wird. Die Schule gewährt ihren Individuen nicht mehr automatisch ihre
Positionen als Schülersubjekte: Sie müssen erarbeitet werden und können jederzeit wieder entzogen werden. Dementsprechend werden schulische Bedingungen von Subjektivierung unter stärkerer Akzentuierung des Leistungsprinzips geschaffen, d. h. an messbaren, sichtbaren Leistungsprodukten.

Hier wird klar, dass sich die auszubildenden charakterlichen Eigenschaften sehr stark an ökonomischen Kategorien orientieren. In dieser Deutung fällt den persönlichen Eigenschaften eine hohe Bedeutung bei der Organisation (wissens-)ökonomischer Arbeitsverhältnisse $\mathrm{zu}$, die in hohem Maße von Flexibilität, Kreativität und Selbstorganisation abhängig sein würden. In diesem Diskurs wird auch die Bedeutung des lebenslangen Lernens betont, für die nicht zuletzt charakterliche Eigenschaften, wie Bereitschaft und Freude an Weiterbildungsprozessen, ausgebildet werden sollen. Die Aussage der ehemaligen Ministerin Schmied muss dementsprechend so gedeutet werden, dass es zur essenziellen Aufgabe von Schule wird, die Beschäftigungsfähigkeit von SchülerInnen zu gewährleisten und dafür bestimmte persönliche und charakterliche Eigenschaften und Fähigkeiten zu vermitteln, ebenso wie Bereitschaft zu und Freude an Weiterbildungsprozessen. Freude am Lernen zu haben, ist dann nicht mehr ein Selbstzweck, den die Schule fördern soll, sondern zahlt sich wie eine Investition langfristig aus, da sie qualifikatorische Vorteile am Arbeitsmarkt bedeutet.

Eine unmittelbare Folge dessen ist, dass sich die Entgrenzung von Lernen ebenso auf das Produkt von Lernen bezieht. Aussagen, die eine geringer werdende Bedeutung von standardisierten Leistungen (wie auch vom Verfahren der standardisierten Leistungsbeurteilung insgesamt) argumentieren, sind nicht als Verzicht auf das Leistungsprinzip, sondern im Gegenteil als Bedingung zu dessen Ausdehnung zu interpretieren: Sie folgen der Annahme, dass Leistung auf vielfältige Art erbracht werden kann. Das Lernprodukt soll sich dem Lernenden anpassen, denn dies erhöht die Anforderungen des Individuums auf Nutzung seiner personalen Eigenschaften, besonders von Kreativität und Techniken der Selbstorganisation. Eine breite Vielfalt möglicher Leistungsprodukte ermöglicht eine zielgerichtete, strategische Ausbildung individueller Dispositionen und Fähigkeiten. Mit der Erweiterung von möglichen Leistungsprodukten verstärkt sich somit auch der Aufforderungscharakter, eine dem Individuum möglichst entsprechende Leistung zu erbringen: Lernleistungen sollen nun besonders kreativ, sollen 
Ausdruck der individuellen Persönlichkeit des Lernenden sein. Hier klingt auch die Idee an, dass Lernen Freude machen kann und soll: Die Freiheit, ein Lernprodukt der eigenen Wahl zu gestalten, soll genutzt werden, und es gibt keinen Grund mehr, dies negativ zu sehen - Lernprodukte sollen individueller, kreativer, und freudvoller Ausdruck der eigenen Beschäftigung mit dem Lerngegenstand sein.

\section{Praktiken des Lernens und dominante Ideologien}

In diesem Abschnitt wird nun letztlich analysiert, welche existierenden gesellschaftlichen Ideologien in Lernpraktiken artikuliert, aktualisiert und vermittelt werden, d. h. es wird erörtert, wie die bestehende Praxis des Lernens ideologische Deutungen über die Struktur der gegenwärtigen Gesellschaft stützt. Dabei soll immer zunächst von Praxisformen des Lernens ausgegangen werden und ihre spezifischen Verzerrungen durch Ideologie, um dann dominante Deutungen herauszuarbeiten, die durch diese Praktiken erworben werden.

These I: Individualisierte Lernpraktiken vermitteln die Machttechnik gouvernementaler Selbstführung.

Bereits festgehalten wurde, dass viele Praktiken der Individualisierungsdidaktik im Diskurs als Maßnahmen zur Leistungssteigerung positioniert wurden. Besonders durch eine Verbindung zu bildungsökonomischen Aussagen, wie sie in der diskursiven Formation des lebenslangen Lernens entstanden ist, wurde die individuelle Nutzbarmachung persönlicher Ressourcen postuliert. Dabei ist hier nochmals festzuhalten, dass individualisiertes Lernen ebenso ohne eine ökonomische Dimension, also jenseits der Frage des langfristigen Nutzens, als Prozess der Selbstbildung und des Ausbaus individueller Stärken und Fähigkeiten vorstellbar wäre. So ist die hier beschriebene Funktionalisierung zu verstehen - die inzwischen scheinbar selbstverständliche Ausrichtung von Lernprozessen hin zu einem beruflichen oder anders monetär „sinnvollen“ Ziel wird im pädagogischen Konzept der Individualisierung nicht expliziert.

Diese Funktionalisierung bedeutet nun - wie ebenfalls schon erwähnt wurde -, dass es keine a priori definierten Lernziele mehr geben kann; der Lernerfolg zeigt sich für das Subjekt vielmehr in dessen Bestehen am Arbeitsmarkt. Damit wird den Subjekten (allgemein gesprochen) nahegelegt, unter Perspektiven von (subjektiv angenommenen) ökonomischen Möglichkeiten zu handeln; eigene Fähigkeiten und Wünsche analog zu ökonomischen Anforderungen zu entwickeln. Hier wird also ein Moment der Anrufung sichtbar, das darin besteht, sich als Lernsubjekt um die ökonomische Nutzbarmachung der eigenen Individualität zu bemühen.

Ähnliches gilt für weitere, mit Individualisierung assoziierte Praktiken des Lernens: Kreativität, Eigenverantwortung, Chancengerechtigkeit, Lebensnähe und Effizienz als zentrale Parameter eines gelungenen Lernprozesses. All diese Momente verfügen über keine a priori definierte Bedeutung, die auf ökonomischstrategisches Handeln verweist. In der diskursiven Formation, in welche die Schule nun eingebunden ist, werden diese Elemente jedoch so angeordnet, dass sie eine ideologische Funktion einnehmen. Ihre konkrete Bedeutung wird dem Individuum in seiner Anrufung als Lernsubjekt nahegelegt und durch die richtige, ökonomisch nachhaltige Anwendung im Lernprozess selbst aktualisiert. Auch die Frage von Chancengerechtigkeit im Lernen wurde nicht nur ökonomisch funktionalisiert, indem sie das Leistungsprinzip stabilisiert, sondern erhält ebenso ein ideologisches Moment der Anrufung, indem SchülerInnen dazu aufgefordert werden, am Wettbewerb teilzunehmen - nun, da alle diskriminierenden Faktoren beseitigt wären.

Individualisiertes Lernen ist hierbei aber das zentrale Deutungselement, weil es die für die gegenwärtige kapitalistische Ökonomie so bedeutsame Unbestimmtheit verdeutlicht, welche die Machtform gouvernementaler Führung charakterisiert. Statt klarer Vorgaben und Regulierungen legitimiert sich der Lernfortschritt des Subjekts allein an dessen Beschäftigungsfähigkeit und dessen Bestehen im Wettbewerb; somit stellt das Scheitern ein sehr viel bedrohlicheres Szenario dar als der innerinstitutionelle Regelverstoß. Mögliche Konsequenzen des Scheiterns sind existentieller Natur, sie sind keine kalkulierbare Sanktion der Institution mehr. Vielmehr sind sie wenig vorhersehbar und verfügen dazu über einen naturhaften Charakter, da der Arbeitsmarkt im Diskurs selbst oft als fetischhaft (im Marx'schen Sinn) beschrieben wird.

Individualisiertes Lernen als Praktik, die im Modus gouvernementaler Selbstführung ausgeübt wird, bedeutet dann, dass Subjekte Lernen als entgrenzten, lebenslangen Prozess wahrnehmen, in welchem eigene Potenziale gezielt genutzt und Leistungen erbracht werden sollen. Welche das sind, wird ebenso wie die Wahl der Demonstrationsmöglichkeiten (d. h. der erzeugten Lernprodukte) und die Verantwortung über den Lernerfolg dem/der Lernenden selbst über- 
lassen. Weil sich aber Sinn und Nutzen eines jeden Lernprozesses erst im Verlauf der individuellen Biografie zeigen und nicht vorher definierbar sind, könnte ein jeder Lernprozess auch potenziell sinnvoll sein, ist jede Anstrengung wichtig und notwendig und ist jedes Scheitern als ein Zuwenig dieser Anstrengung zu deuten.

Praktiken der Individualisierung sind daher als Prozesse der permanenten individuellen Subjektwerdung $\mathrm{zu}$ interpretieren, die unter der Maxime der erschöpfenden Ausnutzung aller Stärken, Möglichkeiten und Potenziale Gültigkeit finden, aber in der Zurückweisung aller vorgefertigten inhaltlichen Verläufe. Entgrenzung bedeutet dabei zugleich eine Zurückweisung jeder inhaltlichen Vorgabe wie auch eine Maximierung aller Anstrengung, denn die einzigen Grenzen setzt sich der/die Lernende selbst. Denn die Individualität gilt es dabei nicht nur auszubilden, sondern auch (sozial) abzusichern; beide Prozesse sind jedoch niemals abgeschlossen. Die Übertragung ökonomischer Deutungen auf Lernen bedeutet dabei, dass ähnlich wie in der kapitalistischen Ökonomie Stagnation zugleich Abstieg im Wettbewerb bedeutet. Analog zur kapitalistischen Wachstumsideologie bedeutet erfolgreiches Lernen einen Zuwachs des akkumulierten Wissens, das durch Weiterbildungsmaßnahmen immer noch gesteigert werden könnte. Das Moment der Übertragung dieser Denkweise führt zur zweiten These.

These II: Die zentralen ideologischen Momente konstituieren einen selbstreferenziellen Rahmen vernünftigen Handelns und generieren verbindliche Handlungsmaximen.

Für die Subjektivierungsform „des unternehmerischen Selbst“ hat Bröckling (2007) darauf hingewiesen, dass in dessen Zentrum privilegierte Signifikanten eine fixierte Bedeutung einnehmen müssen, damit das Subjekt sein Leben eigenverantwortlich gestalten kann. Sie stellen ein Handlungsschema dar, d. h. Maximen, die weitere Tätigkeiten und Handlungen strukturieren. Für Bröckling sind das besonders das Gebot der Selbstoptimierung, der Effizienzsteigerung und Profitmaximierung. Sie erst konturieren die Bedeutung weiterer Äquivalente. Ohne dieses feste Raster, das für alle Subjekte verbindlich ist, gäbe es keine Konkurrenz und keinen unternehmerischen Wettbewerb, der Machtwirkung auf das Handeln der Subjekte entfaltet. Bröckling (2007: 107) greift dabei auf den Begriff der Rationalität zurück und meint damit eine „Ratio neoliberalen Regierens", die in der neoliberalen Ideologie postuliert wird, aber letztlich unerreichbar ist. Die Pointe neoliberaler Ratio besteht darin, dass sie vom Subjekt ein gewisses Verhalten einfordert, das sich nicht auszahlen kann, wenn sich alle Subjekte so verhalten würden: „Rational zu handeln, ist demnach gleichermaßen universelle Intention wie unerreichbare Zielmarke." (ebenda: 143) Entscheidend für die hier unternommenen Überlegungen zur Ideologie ist aber, dass eine spezifische Handlungsweise von Subjekten als vernünftig angenommen wird und sie über diese Positionierung als „vernünftig“ eine große Machtwirkung entfaltet.

Der Begriff der Rationalität, auf den Bröckling rekurriert, ist womöglich problematisch, da er ein a priori von Bedeutungen unterstellt, das von den Subjekten scheinbar unabhängig und selbstreferenziell existieren kann. Doch wird der Begriff nicht so verstanden, denn für Bröckling ist entscheidend, dass es „die“ Rationalität nicht gibt, sondern die Ideologie eine ganz bestimmte Handlungsmaxime diskursiv als vernünftig positioniert. Was Bröckling als Rationalität bezeichnet, ist eine fixe Äquivalenzkette, die sich um privilegierte Signifikanten gebildet hat und die tatsächlich selbstreferenziell, als in sich geschlossenes System existiert. Die privilegierten Signifikanten - in den Praktiken des Lernens sind das die Signifikanten Eigenverantwortung, Individualisierung, Entgrenzung, Vergleichbarkeit und Leistung - konstituieren ihre Bedeutung durch eine relationale Fixierung zueinander und wirken dadurch strukturierend auf Elemente der Lernpraxis. Weil also beispielsweise der Signifikant „Eigenverantwortung“ im Zusammenhang zum Signifikanten „Entgrenzung“ positioniert wird, erhalten beide eine verbindliche Bedeutung und können dadurch die Deutung von weiteren Äquivalenten in Lernpraktiken fixieren.

Die zentrale ideologische Wirkung besteht in dieser Naturalisierung von Handlungsmaximen, die aus der Position in der Äquivalenzkette entsteht: Die Bedeutung von Elementen wie „Freude am Lernen“ (siehe These III) wird in Abhängigkeit zu privilegierten Signifikanten bestimmt und daher im Sinn von hegemonialen Deutungsmustern des idealen Lernens. Damit lassen sie einen spezifischen Umgang mit diesen Elementen als vernünftig erscheinen. Für Praktiken gouvernementaler Selbstführung ist das essenziell: Das Schülersubjekt soll dahingehend funktionieren, dass es selbstständig, eigenverantwortlich und mit größtmöglicher Effizienz lernt, ohne dass klare Regeln, Vorgaben und Abläufe bereitgestellt werden. Als Schülersubjekt eigenverantwortlich zu lernen, setzt die Beherrschung der Logiken und Erkenntnisweisen eines idealen Lern- 
vorgangs voraus. Es soll selbst erkennen, welche Handlungsweisen im Sinne der Deutung des idealen Lernens naheliegen und diese anwenden, $d$. h. welches Handeln in diesem Sinne „vernünftig“ ist. Es bedarf dann keiner guten Argumente oder Rechtfertigungen, wenn etwa eine Lehrperson eine/n Schüler/in auffordert, motiviert, kreativ o. ä. zu sein oder in Eigenverantwortung zu lernen - es bedarf vielmehr einer Rechtfertigung, das nicht zu tun.

An Bröckling anknüpfend soll hier argumentiert werden, dass die verzerrenden ideologischen Operationen in Deutungen des idealen Lernens in ihrer relationalen Beziehung zueinander auftreten, d. h. als ein Set von stabilisierten Bedeutungen. Weil die Schule die "richtige“ Durchführung von Praktiken belohnt und der/die SchülerIn dadurch als erfolgreiches Schülersubjekt gilt, erscheint ihm/ihr diese Art der Anwendung als vernünftig. Die Deutung, welches Handeln als vernünftig gilt, wird über konkrete Praktiken in der Schule konstituiert und entfaltet zugleich über Prozesse der Anrufung, ein vernünftig handelndes Schülersubjekt zu sein, ihre Wirkungsmacht. Die dritte These beschreibt die daraus resultierende Wirkung auf Subjektivierungsvorgänge für das Miteinander und die Rolle des/der Einzelnen im Klassenzimmer.

These III: Die ökonomische Funktionalisierung erzeugt Widersprüchlichkeiten in damit angebotenen Formen von Subjektivität.

Ausgangspunkt dieser These sind die Analysen von Hardt und Negri (2005: 100f.), die Schule als Ort der biopolitischen Produktion erkennen. Hardt und Negri erklären die steigende Bedeutung persönlicher Eigenschaften mit der Hegemonie „immaterieller Arbeit“ als gesellschaftlicher Produktionsform; wie auch der Kapitalismus zu einer Ausdehnung des Bereichs der Produktion führt, die sich nicht mehr auf die Arbeit beschränkt. Im gegenwärtigen Kapitalismus werden nicht nur Formen von Erwerbsarbeit, sondern auch Formen der Freizeit und Reproduktionsarbeit mitproduziert. Der Begriff „biopolitisch“ bedeutet, dass immaterielle Arbeit eine Unterwerfung persönlicher, aber auch körperlicher Dimensionen erfordert - die Regulierung und Nutzbarmachung von Kreativität, Mobilität, Gefühlen und Affekten. Im ökonomischen Produktionsprozess lässt sich so zusehends keine klare Trennung zwischen gesellschaftlicher und privater Sphäre mehr feststellen, wie auch in den „creative industries“ eine Trennung zwischen Freizeit und Arbeitszeit zunehmend aufgehoben wird. Hardt und Negri (2002: 33of.) beschreiben, wie sich als Folge dessen eine „hybride Konstitution“ von Subjektivität, zwischen den Sphären der Produktion und Konsumption, zwischen privater und gesellschaftlicher Felder, vollzieht.

Aus dem geschilderten Individualisierungsimperativ im Hinblick auf die Arbeitswelt scheint klar, dass die Schule keine eindeutigen Subjektpositionen bereitstellt; sondern vielmehr, dass es zur Herausforderung des Schülersubjekts wird, innerhalb der Bandbreite möglicher Subjektpositionen seine/ihre eigene zu bestimmen und zu erarbeiten. Jedoch zeigen sich im Prozess der Subjektivierung zwei widersprüchliche Kraftfelder, die Widersprüche in möglichen Formen von Subjektivität erzeugen.

Der Widerspruch entsteht, weil mögliche Formen von Subjektivität entlang des Signifikanten des ökonomischen Vorteils konstituiert werden, wobei dieser Signifikant leer und nicht eindeutig bestimmbar ist. Über eine Antwort auf die Frage, was, wie und zu welchem Ziel gelernt werden soll, verfügt das Individuum zum Zeitpunkt des Lernens demnach nicht - es kann nur auf verschiedene existierende Aussagen und Annahmen zurückgreifen und dahingehend handeln. Die Herausforderung für das Subjekt besteht besonders darin, eine passende Form von Subjektivität zwischen Individualisierung und Anpassung, zwischen eigenen Bedürfnissen und Anforderungen des Marktes, aber besonders zwischen sozialem Handeln und eigenem Erfolgsstreben anzunehmen - denn diese Subjektivitätsformen sind teils widersprüchlich.

Einerseits zeigt sich eine Tendenz, die eine charakterliche Homogenisierung der Schülersubjekte im Zuge ihrer personalen Selbstbildung bewirkt. Die (diskursiv vollzogene) Normierung eines Repertoires erwünschter Eigenschaften verfügt über eine ausschließende Wirkung für jene Charaktereigenschaften und Gewohnheiten, die als inkompatibel gedeutet werden, um im Kapitalismus Erfolg zu haben. Umgekehrt gelten gewisse Fähigkeiten und charakterliche Eigenschaften als nötig, um karrieregünstige Lebensverläufe und prestigereiche Positionen $\mathrm{zu}$ erreichen. Dieses Repertoire an personalen Merkmalen ist jedoch keineswegs absolut, sondern wandelbar - diese letztliche Unbestimmbarkeit erhöht wiederum den Charakter gouvernementaler Selbstführung als Form der Machtausübung in der Schule und der Orientierung an dem Leitbild des , unternehmerischen Selbst“.

Dabei spricht vieles dafür, dass die Reichweite (im Sinne ihres Einflusses) diskursiver Leitbilder auf gegenwärtige Subjektivitätsformen deutlich höher ist, 
als es Bröckling mit dem unternehmerischen Selbst beschreibt - in dem Sinne, dass persönliche Bereiche noch stärker umfasst werden, als dies Bröckling annimmt. Als Beispiel dafür kann die steigende Anzahl persönlicher Blogs ${ }^{1}$ herangezogen werden: Persönliche Blogs, die von Menschen ohne spezifische Ziele außer dem Erfolg dieser Blogs betrieben werden, thematisieren die eigene Lebensführung in Praktiken wie Kochen, Bekleidung, Sport- und Freizeitverhalten. Ohne dass gesicherte empirische Evidenz über die Wirkung dieser Blogs vorliegt, kann angenommen werden, dass ihnen eine Leitbildfunktion für die Organisation von alltäglichen Praktiken zukommt. Dafür spricht nicht nur ihr Effekt einer erhöhten Thematisierung von Praktiken im privaten Bereich wie auch der leichteren Zugänglichkeit über das Internet, sondern besonders der ökonomische Erfolg dieser Blogs und ihrer Finanzierung durch Produktwerbung von Firmen, die auf die Wirkung dieser Blogs bauen. Je mehr Leitbilder im Feld der privaten Praktiken entstehen und je mehr damit Möglichkeiten ihrer ökonomischen Ausschöpfung geschaffen werden, desto mehr Felder entstehen, innerhalb derer es $\mathrm{zu}$ einer Homogenisierungstendenz von Formen der Subjektivität kommt. Zugleich expandiert damit das Feld möglicher Wissensformen und damit Lerninhalte, da in jedem Feld Profit- und Beschäftigungsmöglichkeiten entstehen, wenn sie dem kapitalistischen Zugriff unterliegen.

Während bislang für eine zunehmende Homogenisierung von Subjektpositionen argumentiert wurde, ist mit der höheren Reichweite von Leitbildern personaler Lebensführung aber auch ein gegenläufiger Effekt festzustellen. Denn mit steigender Zahl und höherer Verbreitung werden die angebotenen Leitbilder beliebiger, oberflächlicher und bedingen eine Erosion universeller, sozial verbindlicher Normen, die sich auf das gesellschaftliche Miteinander beziehen. Denn jene Leitbilder beziehen sich stärker auf die Sphäre des Konsums und postulieren die Austauschbarkeit von Produkten wie auch, dass der eigene Nutzen von Dingen im Vordergrund steht. Dies führt letztlich

1 Gemeint sind dabei Webseiten, die sich mit Fragen „alltäglicher Lebensführung“ im Sinne Holzkamps (1995) befassen und mit dem Anspruch auftreten, dem Individuum bei der Organisation alltäglicher Praktiken unterstützend zur Seite zu stehen. Eine wissenssoziologisch-diskursanalytische Untersuchung dieser Blogs und der hier andiskutierten Frage, inwieweit sich alltägliche Praktiken durch das Aufgreifen dieses Gegenstandes durch Blogs ändern, steht bislang noch aus. dazu, dass auch soziale Beziehungen zusehends warenförmigen Charakter annehmen. Auf diesen Aspekt weist besonders Lessenich (2008) hin, der die These einer „Neuerfindung des Sozialen“ vertritt. Lessenich zufolge verlieren Leitbilder, die am sozialen Miteinander und am Interesse der Gemeinschaft orientiert sind, immer mehr an Deutungskraft gegenüber einer kurzfristigen, den eigenen Nutzen erhöhenden Interaktionsweise, die sich an Verfahrensweisen und Prinzipien der Ökonomie orientiert. Die Diagnose lautet, dass "[...] der flexible Kapitalismus einer projektbasierten Rechtfertigungsordnung [folgt], die alle sozialen Beziehungen in einer auf Anpassungsfähigkeit und Kurzfristigkeit setzenden, netzwerkförmig angelegten Struktur aufgehen lässt [...]“ (Lessenich 2008: 76). Der Wert einer Gemeinschaft (und ihrer Normen) wird vor dem Hintergrund ihres Wohlstandes gemessen; auch das Individuum bestimmt sich durch seinen Nutzen, den es für die gesamte Gesellschaft hat. Die Nutzbarmachung der persönlichen Fähigkeiten eines Individuums wird für Lessenich zur moralischen Verantwortung für die Gesellschaft: „Umgekehrt muss in dieser Konstellation jeder Akt unterlassener Hilfeleistung der Individuen gegenüber sich selbst als nicht nur irrationales, sondern zudem noch unmoralisches Verhalten erscheinen, gilt hier das Anzeichen fehlender oder mangelnder Aktivitätsbereitschaft nicht bloß als unwirtschaftlich, sondern asozial." (ebenda: 83)

Wird der Wert des Individuums aber zusehends an seinem materiellen Wert für die Gemeinschaft gemessen, sinkt die soziale Verbindlichkeit von Normen jenseits dieses Standards wie auch Formen sozialen Zusammenhalts, die sich gerade dadurch kennzeichnen, dass sie auf keiner materiellen Basis stehen. Gemeint sind hierbei Werte, die das eigene Fortkommen womöglich behindern, aber für eine Gemeinschaft konstitutiv sind - Solidarität, Hilfsbereitschaft, Zusammenhalt. Eine ökonomische und individualisierte Subjektivitätsform generiert Handlungsmaximen primär auf Basis des individuellen Nutzens und misst der Gemeinschaft und sozialem Handeln daher keinen Eigenwert bei.

Auch für das Lernen kann diese Tendenz bedeuten, dass die Verbindlichkeit sozialer Normen abgeschwächt werden könnte, wenn sie in einem Widerspruch zur Maxime des ökonomischen Nutzens des Lernens stehen. Sozialen Normen, die in Lernzielen, Lerninhalten und Sozialformen des Lernens vermittelt werden, kommt kein Eigenwert zu, wenn es in der Schule nur noch um den Lernerfolg geht. Zudem beinhaltet die 
Aussage, dass sich Lernen primär an den Bedürfnissen des Individuums orientieren und sich dessen Zwecken unterordnen soll, nichts sozial Verbindliches. Die Maxime des Lernens unter dem Gebot eines zweckbezogenen Individualismus kann wiederum mit Lessenich (2008: 83) so zusammengefasst werden, „dass die Subjekte frei sind, so zu handeln, wie es der liberalen Rationalität entspricht".

Nicht zuletzt formuliert Reichenbach (2014) die These, dass mit einer stärkeren Individualisierung ein Rückzug pädagogischer Verantwortung, mithin eine Transformation des pädagogischen Auftrags selbst, zu beobachten sei. Es spricht einiges dafür, dass die gesellschaftliche Tendenz zur Individualisierung eine solche Heterogenität von Lebensverhältnissen erzeugt hat, dass im Klassenzimmer kein verbindlicher, Wahrnehmungen strukturierender Deutungs- und Interpretationsmaßstab jenseits der eben erwähnten, stark individualistisch bezogenen Auffassung mehr existiert. Jedoch findet diese Interpretation von idealem Lernen bei SchülerInnen selbst nicht nur Zustimmung - und diese Brüche gilt es zu nutzen, um die Ideologie generell zu thematisieren.

These IV: Die ideologische Reproduktion ist kein determinierender, sondern ein dialektischer Vorgang.

Mit der zuletzt formulierten These wird die Frage aufgeworfen, wie Prozesse der Subjektivierung verlaufen. Die letzte These soll betonen, dass Subjektivierung als Prozess der Anleitung zu bestimmten Subjektpositionen zu denken ist, jedoch keineswegs als Determinierung oder Fixierung. Es soll auch darauf hingewiesen werden, dass in und durch diese Anleitung Brüche entstehen, die von Lehrpersonen als emanzipative Auswege genutzt werden können.

Dazu soll zunächst nochmals die Wirkung von Ideologien in der Schule rekapituliert werden. In den vorangegangenen Thesen wurde dafür argumentiert, dass SchülerInnen von der Institution Schule veranlasst werden, Praktiken des Lernens eigenverantwortlich $\mathrm{zu}$ organisieren. Dadurch erfahren sie eine spezifische Form der Machtausübung und übernehmen eine Deutung von vernünftigem Handeln, die stark ökonomisch affiziert ist. Auf dieser Basis wird ein eigenes Wahrnehmungs- und Interpretationsrepertoire der unmittelbaren Umwelt gebildet, aber ihre gesellschaftliche Bedingtheit verkannt. Denn letztlich besteht eine zentrale Funktion der Ideologie in der Naturalisierung der Konstitutionsprozesse von schulischen Praktiken, die selbst immer schon ideologisch ist. Wenn bislang davon ausgegangen wurde, dass Ideologien über
Praktiken vermittelt werden, so sollte nun präzisiert werden, dass diese Vermittlung ihren Ursprung im Konstitutionsprozess der Praktiken findet, denen ein Moment der Verzerrung immanent ist. Wie Laclau (2002: 179) ausführt, ist dabei nicht bedeutsam, wie das „eigentliche“ Objekt der Verzerrung einmal ausgesehen hat, sondern, dass die "verzerrende Operation“ nachweisbar ist, d. h. gezeigt werden kann, worin die Verzerrung besteht. Konstitutiv ist die Verzerrung, da Praktiken immer Formierungsprozessen unterliegen, da es keinen außerideologischen Raum gibt.

Wenn in Lernprozessen SchülerInnen ihre Positionen als Lernsubjekte einnehmen, bedeutet das nicht nur, dass sie zu gewissen Handlungen angehalten werden; es bedeutet auch, dass sie im Zuge der Tätigkeit die Struktur und die Regeln der Schule kennenlernen. Sie lernen, auf welche Art und Weise sie handeln müssen, um sich an diesem Ort zurecht zu finden. Sie lernen die Ordnung des Ortes Schule kennen - im wörtlichen Sinne eines spezifischen Arrangements von Gegenständen wie einer Sitzordnung, wie auch im übertragenen Sinn eines Regelsystems. Dieses verfügt über eine Hierarchie von Fächern oder Bildungsinhalten, eine kategoriale Anordnung in Begriffen wie richtig oder falsch, eine Definition impliziter oder expliziter Verhaltensregeln, wie es auch in anderen formellen wie informellen Weisen darlegt, was möglich, erwünscht oder verboten ist. Die Funktionsweise des lokalen Ortes Schule wird so als strukturierendes Prinzip oder Regelsystem aufgefasst, dessen innere Grammatik von SchülerInnen „intuitiv“ im Alltag erkannt wird. Es ist - in gewisser Weise - das „Hidden Curriculum" von Schule und organisiert die Reproduktion der Gesellschaft durch verschiedene Prozesse der Naturalisierung und Normalisierung. Die Produktion von Ideologie geschieht dabei nicht in der Praxis: Jede Praxis ist bereits ideologisch, da die Reproduktion der Gesellschaft in und durch alle schulischen Praktiken stattfindet.

Nachdem SchülerInnen ihre Lernpraktiken vielfach selbst organisieren und (innerhalb einer definierten Bandbreite von Möglichkeiten) nach freiem Willen ausgestalten können, ist klar, dass der Prozess der Konstitution von Ideologie einer ist, an dem die AkteurInnen von Schule selbst aktiv beteiligt sind. Aktiv meint dabei nicht, dass den Subjekten ihre Rolle immer bewusst ist; verschiedene Prozesse der Naturalisierung und Normalisierung, die dem schulischen Alltag immanent sind, verhindern diese Einsicht oftmals. Aktiv meint jedoch, dass den Subjekten selbst 
eine Einsicht über die Struktur dieser Grammatik und ihrer gesellschaftlichen Bedingtheit zuzutrauen ist, und hieraus können verschiedene Brüche entstehen oder bewusst herbeigeführt werden.

Althusser (2010: 43) hat festgestellt, die Integration des Subjekts über den ideologischen Staatsapparat Schule würde so "geräuschlos“ vonstattengehen. Damit gab er einen entscheidenden Hinweis über die Natur der Brüche: Brüche sind jene konfrontativen, lauten Akte im schulischen Alltag, bei denen sprachlich ausgeschlossene, naturalisierte Modi von Praktiken plötzlich zum Thema gemacht werden. Ideologische Prozesse von Schule - Verfremden, Verkennen, Verzerren basieren darauf, bestimmte Deutungen nicht offen $\mathrm{zu}$ debattieren, sondern mittels Praktiken im Alltag zu normalisieren. Brüche sind jene Momente, in denen die Widersprüchlichkeit, die zwischen Deutungen, die SchülerInnen im Prozess familiärer Sozialisation erfahren haben, und neuen einzunehmenden Subjektpositionen offen zutage treten. Diese Brüche sind als dialektische Momente zu denken: Sie sind Reaktion und Widerstand auf schulische Ordnungen, die die Schule selbst erzeugt beim Versuch, eine bestimmte Ordnung durchzusetzen. Dialektisch ist hierbei im Sinne Hegels zu verstehen: Der positiv-verständigenden These setzt die Schule selbst das negativ-aufhebende Moment gegenüber, indem sie im Subjekt den Widerstand evoziert, den sie auszuschließen sucht.

Subjektivierung als Praktik zu beschreiben, die ohne Zutun des Subjekts geschieht, endet in einem deterministischen Schluss ohne emanzipativen Ausweg. Dies führt zu einer Beschreibung von Subjektivität als „falsches Bewusstsein“, und diese Beschreibung kann jene produktiven Momente gar nicht erkennen, die in der Schule unbeabsichtigt existieren und als Brüche gegenläufige Formen von Subjektivität erzeugen können. Daher muss eine Perspektive eingenommen werden, die danach fragt, inwieweit in der Schule Lernen auf eine subversive Art und Weise stattfinden kann, welche die skizzierte reproduktive Funktion von Lernen unterlaufen kann. Die ideologische Reproduktion ist ein dialektischer und kein determinierender Prozess, der nicht nur Nähe und Affirmation, sondern auch Distanz und Irritation erzeugt. Die Frage ist nun, auf welche Weise diese Brüche genutzt werden können - und wie etwa Lehrpersonen diese nutzen können. Giroux (2012) liefert mit seinem Konzept des „teachers as intellectuals“ dazu wertvolle Hinweise, die an dieser Stelle leider nicht mehr ausgeführt werden können.

\section{Fazit}

Der Beitrag hat die historisch lang rezipierte Frage aufgegriffen, wie in der Schule gesellschaftliche Ideologien vermittelt werden. Dabei wurde im Anschluss an den Ideologiebegriff Althussers angenommen, dass Ideologie als tätige Praxis begriffen werden muss. Die derzeit wirksame Ideologie in der Schule wurde anhand von Leitbildern des idealen Lernens (als der zentralen Tätigkeit der Schülersubjekte) analysiert. In diesem Leitbild wurden einige zentrale Werte identifiziert, die als privilegierte Signifikanten des Diskurses fungieren. Diese stehen im Kern der schulischen Ideologie, sie bilden Deutungsmaximen des vernünftigen Handelns in der Schule. Sie werden vom Schülersubjekt als strukturierendes Prinzip oder Regelsystem erkannt und in der Anrufung als erfolgreiches Subjekt anerkannt. Die Wirkungsweise von Ideologie wurde als gouvernementale Selbstführung beschrieben, die über Praktiken angeleitet wird.

Subjektivierungsvorgänge in der Schule bestehen darin, dass Kinder angeleitet sind, (in einem unternehmerischen Sinn) eigenverantwortliche Lernende zu werden. Anders als in früheren Zeiten setzt die Schule dabei immer weniger auf die Machttechnik der Disziplinierung. Sie versucht vielmehr, über Momente der Anrufung das Individuum dazu zu bewegen, Strukturen und Regeln von selbst zu befolgen. Diesen Mechanismus gouvernementaler Selbstführung hat Foucault (2000) als Machttechnik beschrieben, die aus einer Engführung von Disziplin und Technologien des Selbst zu verstehen sind, und dabei darauf hingewiesen, dass Praktiken und Tätigkeiten als jene Dispositive der Macht fungieren können, über die sich Herrschaftsverhältnisse reproduzieren.

Im Zentrum dieses Artikels stand daher die Analyse von Praktiken, in denen Vermittlungsprozesse von Ideologie identifiziert wurden. Es wurde gezeigt, wie die privilegierten Signifikanten Entgrenzung, Individualisierung, Eigenverantwortung und Kreativität von Lernprodukten Praktiken des Lernens konstituieren, wobei diese Formierung im Anschluss an Laclau als eine Operation ideologischer Verzerrung beschrieben wurde. Diese Deutungen generieren den Rahmen von Lernprozessen und werden über diese Praktiken als Teil des Lernprozesses vermittelt. Die ideologische Operation der Verzerrung von Lernpraktiken findet also unter dem Gesichtspunkt statt, Lernpraktiken auf Basis dieser Deutungen zu gestalten, da in diesen Deutungen selbst Annahmen über die Gesellschaftlich- 
keit des Lernens enthalten sind - etwa, was wichtige Inhalte sind, wozu gelernt werden soll, wie verbindlich Lernleistungen erbracht werden usw. Ihre Folge sind Momente der Verkennung, Abstraktion und Naturalisierung über die Konstitution von Lernpraktiken durch das Subjekt, indem diese gesellschaftliche Dimension bzw. die Funktion des Lernens für gesellschaftliche Reproduktionsprozesse nicht mehr sichtbar wird.

Zugleich ist wichtig, nochmals darauf hinzuweisen, dass in den ideologischen Strukturen der Schule keine präskriptive inhaltliche Dimension angelegt ist. Einerseits, weil die konkrete Ideologie sich aus der Art der Verbindung zwischen verschiedenen Elementen rund um die privilegierten Signifikanten bestimmt und dabei nur temporär fixiert ist. Privilegierte Signifikanten legen die Übernahme von Deutungen und Wahrnehmungsweisen nahe, ohne sie zu determinieren, und zumeist geschieht dies nicht explizit - im Gegenteil, ist in der schulischen Praxis der Betonung von Neutralität und Ausgewogenheit selbst schon eine ideologische Strategie, die hegemoniale Sichtweisen favorisiert. Dass die bewusste Unbestimmtheit oftmals Bedingung für die Vermittlung von Ideologie ist, wird oftmals vergessen. Andererseits, weil die Wirkungsweise von Ideologie als ein grundsätzlich dialektisches Phänomen zu begreifen ist - Ideologie kann im Subjekt auch Widerspruch erzeugen, auch wenn das der eher seltenere und auch nicht intendierte Fall ist. Dennoch wurde die Dialektik der Vermittlung und Aneignung von Ideologie beschrieben: Die Widerstände, die sich in der Schule oft beobachten lassen, sind letztlich Resultate des ideologischen Vermittlungsprozesses selbst.

Innerhalb der Schulpädagogik, aber auch in der Öffentlichkeit wird die Frage nach der Ideologie im Schulsystem sehr gering rezipiert. Der vorliegende Beitrag möchte ein Anlass sein, diese Fragen erneut aufzugreifen. Er möchte an AkteurInnen appellieren, öfter einmal nachzufragen, was eigentlich gemeint ist, wenn in Gesprächen oder öffentlichen Diskursen von Eigenverantwortung, Chancengerechtigkeit und lebenslangem Lernen gesprochen wird - denn allzu oft gelten diese als „ohnehin klar“, als erstrebenswert oder als Wundermittel zur Lösung aller Probleme des Bildungssystems.

\section{Literatur}

Althusser, L. (2010): Ideologie und ideologische Staatsapparate. In: Wolf, O. F. (Hg.): Louis Althusser: Gesammelte Schriften, 1. Halbband. Hamburg: VSA.

Belina, B./Dzudzek, I. (2009): Diskursforschung als Gesellschaftsforschung - Ideologiekritik und Kritische Diskursanalyse. In: Glasze, G./ Mattissek, A. (Hg.): Handbuch Diskurs und Raum. Bielefeld: Transcript, 129-152.

Bernfeld, S. (1973): Sisyphos oder die Grenzen der Erziehung. Frankfurt a. M.: Suhrkamp.

Bowles, S./Gintis, H. (1976): Schooling in Capitalist America: Educational Reform and the Contradictions of Economic Life. New York: Basic Books.

Brandmayr, M. (2015): Die verborgenen Mechanismen politischer Bildung: Zum Verhältnis von Struktur und Inhalt am Beispiel des Basiskonzepts Macht. Momentum Quaterly, 4 (3), 147-160.

Brandmayr, M. (2017): Dispositive des Lernens. Wiesbaden: Springer (im Erscheinen).

Bröckling, U. (2007): Das unternehmerische Selbst. Soziologie einer Subjektivierungsform. Frankfurt a. M.: Suhrkamp.

Bührmann, A./Schneider, W. (2013): Vom ,discursive turn ${ }^{c}$ zum , dispositive turn'? Folgerungen, Herausforderungen und Perspektiven für die Forschungspraxis. In: CabornWengler, J./Hoffarth, B./Kumięga, L. (Hg.): Verortungen des Dispositiv-Begriffs. Wiesbaden: Springer, 21-35.

Bundesministerium für Bildung und Frauen (2015): „Heinisch-Hosek/Hundstorfer: Vierter Bericht zu „Strategie zum lebensbegleitenden Lernen in Österreich“. Online: http://www.ots.at/presseaussendung/OTS_20150630_ OTSo213/heinisch-hosekhundstorfer-vierter-berichtzu-strategie-zum-lebensbegleitenden-lernen-in-oesterreich [15.5.2016].

Bundesministerium für Unterricht, Kunst und Kultur; Bundesministerium für Wissenschaft und Forschung/Bundesministerium für Arbeit, Soziales und Konsumentenschutz/Bundesministerium für Wirtschaft, Familie und Jugend (Hg.) (2011): LLL:2020. Strategiepapier zum lebensbegleitenden Lernen in Österreich. Online: https:// www.bmb.gv.at/ministerium/vp/2011/lllarbeitspapier_ ebook_gross_20916.pdf?4dtiae [15.3.2016].

Dzudzek, I./ Kunze, C./ Wullweber, J. (Hg.) (2012): Diskurs und Hegemonie. Gesellschaftskritische Perspektiven. Bielefeld: Transcript.

Faulstich, P. (2014): Lerndebatten. Bielefeld: Transcript.

Foucault, M. (1978): Dispositive der Macht. Über Sexualität, Wissen und Wahrheit. Berlin: Merve.

Foucault, M. (2000): Die Gouvernementalität. In: Bröckling, U./Krasmann, S./Lemke, T. (Hg.): Gouvernementalität der Gegenwart. Studien zur Ökonomisierung des Sozialen. Frankfurt a. M.: Suhrkamp, 41-68.

Giroux, H. (2012): The War Against Teachers as Public Intellectuals in Dark Times. Truthout. Online: http:// truth-out.org/opinion/item/13367-the-corporate-war- 
against-teachers-as-public-intellectuals-in-dark-times [22.9.2016].

Glasze, G./Mattissek, A. (2009): Die Hegemonie- und Diskurstheorie von Laclau und Mouffe. In: Dies. (Hg.): Handbuch Diskurs und Raum. Münster: Transcript, 153-179.

Gramsci, A. (1999): Gefängnishefte Band 4. Berlin, Hamburg: Argument.

Hardt, M./Negri, A. (2002): Empire. Die neue Weltordnung. Frankfurt a. M.: Campus.

Hardt, M./Negri, A. (2005): Multitude. War and Democracy in the Age of Empire. New York: Penguin.

Holzkamp, K. (1995): Alltägliche Lebensführung als subjektwissenschaftliches Grundkonzept. Das Argument, 37 (212), 817-846.

Jäger, S. (2000): Theoretische und methodische Aspekte einer Kritischen Diskurs- und Dispositivanalyse. Online: http://www.diss-duisburg.de/Internetbibliothek/ Artikel/Aspekte_einer_Kritischen_Diskursanalyse.htm [22.9.2016].

Jäger, S. (2009): Kritische Diskursanalyse. Eine Einführung. Münster: Unirast.

Keller, R. (2008): Diskurse und Dispositive analysieren: Die wissenssoziologische Diskursanalyse als Beitrag zu einer wissensanalytischen Profilierung der Diskursforschung. Historical Social Research, 33 (1), 73-107.

Keller, R. (2011): Wissenssoziologische Diskursanalyse. Grundlegung eines Forschungsprogramms. Wiesbaden: Springer.

Keller, R. (2014): Wissenssoziologische Diskursforschung und Deutungsmusteranalyse. In: Behnke, C./Lengersdorf, D./Scholz, S. (Hg.): Wissen - Methode - Geschlecht. Wiesbaden: Springer, 143-159.

Kreisky, E. (2002): Ideologie und Ideologiekritik. Thesen, Themen und Materialien zur siebten Vorlesungseinheit vom 21.11.2002. Online: http://evakreisky.at/onlinetexte/ nachlese_ideologie_ideologiekritik.php [22.9.2016].

Laclau, E./Mouffe, C. (1991): Hegemonie und radikale Demokratie. Zur Dekonstruktion des Marxismus. Wien: Passagen Verlag.

Laclau, E. (2002): Tod und Wiederauferstehung der Ideologietheorie. In: Ders.: Emanzipation und Differenz. Wien/ Berlin: Turia+Kant.

Lessenich, S. (2008): Die Neuerfindung des Sozialen. Der Sozialstaat im flexiblen Kapitalismus. Bielefeld: Transcript.

Lukács, G. (1923/1988): Geschichte und Klassenbewusstsein. Darmstadt: Hermann Luchterhand Verlag.

Marx, K./Engels, F. (1958): Die deutsche Ideologie. In: Marx Engels - Werke, Band 3. Berlin: Dietz.

Merkens, A. (2003): Ideologiekritik, Dekonstruktion und Wahrheit. In: Herzfeldt, H./Schäfgen, K. (Hg.): Ferienakademie-Reader der Rosa Luxemburg Stiftung. Berlin: Dietz, 52-66.

Neckel, S. (2010): Refeudalisierung der Ökonomie. Zum Strukturwandel kapitalistischer Wirtschaft. MPIfG Working
Paper 10/6. Online: http://www.mpifg.de/pu/workpap/ wp10-6.pdf [23.2.2016].

Reichenbach, R. (2014): „Progressiv sein heisst heute Dinge konservieren“. Die Wochenzeitung, 8 (2014). Online: http://www.woz.ch/-4bab [23.10.2016].

Rehmann, J. (2004): Ideologietheorie. In: Haug, W.F. (Hg.): Historisch-kritisches Wörterbuch des Marxismus Band 6.I. Berlin: Argument, 717-760.

Schmied, C. (2008): „Schule im Aufbruch“. ILS-Mail 1/2008, 14-15. Online: http://www.uibk.ac.at/ils/ilsmail/pdf_ils_ mail/schule_im_umbruch_online.pdf [5.3.2015].

Sennett, R. (2005): Die neue Kultur des Kapitalismus. Berlin: Berlin Verlag.

Schulqualität Allgemeinbildung (SQA) (2012): Über das Lernen. Leittext der Sektion I (Allgemeinbildung) des BMBF. Online: http://www.sqa.at/pluginfile.php/806/course/ section/352/\%C3\%9Cber\%2odas\%2oLernen_20160706. pdf [15.3.2016].

Willis, P. (1979/2013): Spaß am Widerstand. Gegenkultur in der Arbeiterschule. Hamburg: Argument.

Wexler, P.(1999): Die Toyota-Schule. Ökonomisierung von Bildung und Postmodernes Selbst. In: Sünker, H./Krüger, H.-H. (Hg.): Kritische Erziehungswissenschaft am Neubeginn? Frankfurt a. M.: Suhrkamp, 35-58.

Wolff, R (2004): Ideologischer Staatsapparat/repressiver Staatsapparat. In: Haug, W. F. (Hg.): Historisch-Kritisches Wörterbuch des Marxismus, Band 6.I. Berlin, Hamburg: Argument, 761-772. 